intolerance in association with liver disease is well known and galactose challenge has been used as a dynamic test of liver function. ${ }^{3}$ Pronounced galactose intolerance, however, is usually associated with severe liver disease. ${ }^{2}$ Hepatosplenomegaly was not noted in this infant and biochemical evidence was limited to moderate transient abnormalities of alkaline phosphatase, $\gamma$-glutamyltransferase, albumin, and a persisting borderline rise of transaminase activities. Early jaundice and anaemia are both features of galactosaemia; however, the jaundice was transient, and both prematurity and frequent blood sampling may have been contributory factors.

This child appears to be unusual in the degree of his intolerance to galactose in the absence of any detectable defect in galactose pathways and in the presence of only minimal biochemical evidence of liver disease. This intolerance appeared to be physiological as well as biochemical as the child's health improved promptly when he was given a galactose free diet. It appears unlikely that the biochemical and clinical improvements were unrelated. It is important that children with galactose intolerance other than that due to inherited enzyme deficiencies be challenged with galactose at intervals so that they can return to normal feeds as soon as they are able to tolerate them.

Quantitative assays of erythrocyte enzymes and of galactose-1phosphate were performed by the department of clinical chemistry, Southmead Hospital, Bristol.

\section{References}

1 Schwarz V, Wells AR, Hotzel A, et al. A study of the genetics of galactosaemia. Ann Hum Genet 1961;25:179-88.

2 Wharton BA, Scott PH. The newborn. In: Clayton BE, Round $\mathrm{JM}$, eds. Chemical pathology and the sick child. Oxford and London: Blackwell, 1984:7-72.

${ }^{3}$ Relander A. Intravenous galactose tolerance test. Normal series of children. Scand J Clin Lab Invest 1968;22:196-8.

${ }^{4}$ Segal S. Disorders of galactose metabolism. In: Stanbury JB, Wyngaarden JB, Fredrickson DS, Golstein JL, Brown MS, eds. The metabolic basis of inherited disease. 5th ed. New York: McGraw-Hill, 1983:167-91.

5 Kelly S. Significance of the Duarte/classical galactosaemia genetic compound. J Pediatr 1979;96:937-40.

Correspondence to Dr CJ Taylor, Department of Paediatrics, Children's Hospital, Western Bank, Sheffield S10 2TH.

\title{
Oral vancomycin in prevention of necrotising enterocolitis
}

\author{
P C NG, P R F DEAR, AND D F M THOMAS \\ Department of Paediatrics and Child Health, St James's University Hospital, Leeds
}

\begin{abstract}
SUMMARY Eighty four very low birthweight babies (considered high risk for developing necrotising enterocolitis) were given vancomycin orally for $\mathbf{4 8}$ hours before introduction of oral feeds; one developed necrotising enterocolitis. One hundred and twenty very low birthweight babies (not considered such high risk) were fed without first receiving vancomycin; 17 developed necrotising enterocolitis. Although this was not a randomised control trial, results indicate a role for vancomycin in prophylaxis of necrotising enterocolitis.
\end{abstract}

The exact sequence of pathological events in necrotising enterocolitis has not been established, although there is general agreement that prematurity and neonatal illness predispose to it, and that invasion of the bowel wall by bacteria is the ultimate and most serious aspect of the pathological process. It is also widely accepted that necrotising enterocolitis rarely develops in babies who have not received enteral feeds and indeed often manifests soon after feeds have been introduced. It could be that gut bacteria require food substrate in order to multiply sufficiently to invade the susceptible bowel wall. If this hypothesis is correct, then reducing the bacterial population in the bowel, particularly the gas producing anaerobes, before introducing milk feeds could reduce the incidence of necrotising enterocolitis.

According to this line of reasoning it has been our policy, during the past three years, to treat very low birthweight babies who have had prolonged intensive care, and are therefore at high risk of developing necrotising enterocolitis, with oral vancomycin for $\mathbf{4 8}$ hours before starting milk feeds. Vancomycin was chosen because of its activity against clostridial species and other anaerobes, and because it does not completely sterilise the bowel, which could promote colonisation by unwanted pathogens. Furthermore, vancomycin is poorly absorbed and so a high drug concentration can be achieved in the gut with minimal risk of systemic toxicity.

This paper presents our experience with necrotis- 
ing enterocolitis during the three years that we have used vancomycin prophylaxis.

\section{Patients and methods}

During the three consecutive years from January 1984 to December 1986, 204 very low birthweight babies were admitted to our neonatal unit.

The decision to use vancomycin in a particular case was made neither by defined criteria nor by randomisation, but rather by a consensus of clinical opinion that the baby was at risk of necrotising enterocolitis because of the severity of previous illness. Accordingly, 84 babies were given oral vancomycin for 48 hours before the start of enteral feeding and 120 babies did not receive the drug. Vancomycin was given in doses of $15 \mathrm{mg} / \mathrm{kg}$ eight hourly, for six doses.

Milk feeds were given as intermittent boluses, starting at $1 \mathrm{ml}$ hourly and gradually increasing according to tolerance. Babies were fed human milk whenever possible but a number of commercial formulas were also used.

With regard to outcome, babies were diagnosed as having necrotising entercolitis if they had an illness characterised by abdominal distension, blood in the stools, and intramural gas on abdominal radiography.

We used unpaired $t$ tests for analysis of parametric data, and the Mann-Whitney $U$ test for nonparametric data.

\section{Results}

A total of 28 babies in the group who did not receive vancomycin died within the first 5 days of life before oral feeds were considered. None of them had necrotising enterocolitis. They have been excluded from the analysis, leaving 92 babies in this group.

Table 1 compares the characteristics and occur- rence of necrotising enterocolitis between the groups receiving or not receiving vancomycin treatment. The babies selected to receive vancomycin were, as expected, significantly less mature, smaller, in poorer condition at birth, ventilated for longer, and were more likely to have had an umbilical catheter. They were also significantly older when enteral feeds were first introduced. This is to be expected among a selected high risk group as it is the policy of the unit to delay enteral feeds until babies are free of major respiratory symptoms. A standard parenteral nutrition regime was used until then.

Eighteen cases of definite necrotising enterocolitis were seen during the study period. Seventeen infants were in the group not receiving vancomycin and only one infant was in the group receiving vancomycin (table 2 ). This difference was significant $\left(\chi^{2}=12 \cdot 47, \mathrm{p}<0 \cdot 001\right)$. In the group not receiving vancomycin, three babies died of necrotising enterocolitis. The baby in the group receiving vancomycin underwent surgery but survived. The cases of necrotising enterocolitis were evenly spread throughout the study period and there was no outbreak noted. There was no association between the occurrence of necrotising enterocolitis and the type of milk fed.

Certain clinical features of the 17 babies in the group not receiving vancomycin who developed necrotising enterocolitis were significantly different compared with the 75 babies not receiving vancomycin who did not suffer necrotising enterocolitis (table 2). Those who suffered necrotising enterocolitis had clinical features that were in fact more similar to the group receiving vancomycin, differing significantly only in respect of the later age at which the vancomycin group were first enterally fed.

\section{Discussion}

Considering that infection is undoubtedly an impor-

Table 1 Comparison of characteristics between the two groups. ${ }^{*}$ Results are mean (SD) and range

\begin{tabular}{|c|c|c|c|}
\hline \multirow[b]{2}{*}{ Gestation (weeks) } & $\begin{array}{l}\text { Group receiving } \\
\text { vancomycin }\end{array}$ & \multicolumn{2}{|c|}{$\begin{array}{l}\text { Group not receiving } \\
\text { vancomycin }\end{array}$} \\
\hline & $28 \cdot 5(2 \cdot 4) \quad 24-34$ & $29.8(2.5)$ & $25-35$ \\
\hline Birth weight $(\mathrm{g})$ & 1100 (251) $630-1500$ & 1204 (205) & $625-1500$ \\
\hline Duration of ventilation (days) & $24.5(26.5) 0-114$ & $5.7(10 \cdot 4)$ & $0-63$ \\
\hline Apgar score at 1 minute & $5 \quad 0-10$ & 6 & $1-10$ \\
\hline Apgar score at 5 minutes & $1-10$ & 9 & 4-10 \\
\hline Age when enteral feeds introduced (days) & $24.7(19.8) 2-97$ & $6 \cdot 8 \quad(6 \cdot 2)$ & $1-40$ \\
\hline Incidence of umbilical artery catheterisation (\%) & 82 & & 66 \\
\hline Incidence of necrotising enterocolitis & \multirow{2}{*}{$\begin{array}{l}1 \text { case } \\
55\end{array}$} & \multicolumn{2}{|c|}{17 cases } \\
\hline Age at onset of necrotising enterocolitis (days) & & $19 \cdot 8$ & $(3-37)$ \\
\hline
\end{tabular}

*There was a significant difference (at the $\mathrm{p}<0.02$ level) between the treated and non-treated babies for all the characteristics shown in this table. 
Table 2 Comparison between babies with and without necrotising enterocolitis in the group not receiving vancomycin.* Results are mean (SD) and range

\begin{tabular}{|c|c|c|c|c|}
\hline \multirow{8}{*}{$\begin{array}{l}\text { Gestation (weeks) } \\
\text { Birth weight (g) } \\
\text { Duration of ventilation (days) } \\
\text { Apgar score at } 1 \text { minute } \\
\text { Apgar score at } 5 \text { minutes } \\
\text { Age when enteral feeds introduced (days) } \\
\text { Incidence of umbilical artery catheterisation (\%) }\end{array}$} & \multicolumn{2}{|c|}{$\begin{array}{l}\text { Babies with } \\
\text { necrotising enterocolitis }\end{array}$} & \multicolumn{2}{|c|}{$\begin{array}{l}\text { Babies without } \\
\text { necrotising enterocolitis }\end{array}$} \\
\hline & $28.4(1.8)$ & $26-32$ & $30 \cdot 2(2 \cdot 6)$ & $25-35$ \\
\hline & 1114 (193) & $860-1400$ & 1269 (198) & $625-1500$ \\
\hline & $13.9(17.5)$ & $0-63$ & $3.9 \quad(7.1)$ & $0-54$ \\
\hline & 5 & $1-8$ & 6 & $1-10$ \\
\hline & 8 & $7-10$ & 9 & $4-10$ \\
\hline & $10 \quad(5 \cdot 3)$ & $2-21$ & $6 \cdot 2 \quad(6 \cdot 2)$ & $1-40$ \\
\hline & & 88 & & 61 \\
\hline
\end{tabular}

*There was a significant difference (at the $\mathrm{p}<0.03$ level) between the babies who developed necrotising enterocolitis and those who did not for all the characteristics shown in this table.

tant aetiological factor in necrotising enterocolitis, it is surprising that there have been only four controlled trials of antibacterial prophylaxis. ${ }^{1-4}$ Two of these trials used kanamycin, one showing a significant benefit ${ }^{1}$ and the other not, ${ }^{2}$ and two used gentamicin, both purporting to show benefit. In addition there is the uncontrolled observation suggesting that oral vancomycin might have helped to terminate an epidemic of necrotising enterocolitis. ${ }^{5}$ The question of whether necrotising enterocolitis can be prevented by antibacterial agents therefore deserves further examination.

The experience that we report in this paper does not derive from an attempt to conduct an experiment, but is rather a retrospective review of the effects of a change in clinical practice. As no attempt at randomisation was made there was inevitably bias in the allocation to treatment or non-treatment groups. Indeed there was a deliberate effort to treat with vancomycin those babies judged most susceptible to necrotising enterocolitis according to the generally accepted risk criteria, and in this sense selection bias provides an even more rigorous test of the prophylactic value of vancomycin in necrotising enterocolitis. Interestingly, the 17 babies in the group not receiving vancomycin who developed necrotising enterocolitis are not typical of the nontreatment group as a whole. In terms of the putative risk factors - namely, shorter gestation, lower birth weight, poorer condition at birth, and more prolonged ventilation-they were appreciably more at risk than the remainder of the group not receiving vancomycin. In a formal study, the 17 babies in question might have been identified as 'at risk' and therefore treated. Decision making in routine clinical practice is, however, rarely as rigorous as it might be.

While selection bias undoubtedly strengthens the conclusion that something occurring to the group receiving vancomycin resulted in a significant reduc- tion in the expected incidence of necrotising enterocolitis, it prevents us from concluding with certainty that it was vancomycin. A possible alternative explanation is the difference between the two groups in the age at which feeds were introduced. ${ }^{6}$ The group receiving vancomycin were first fed at a mean of 24.7 days of age and the group not receiving vancomycin at a mean of $6 \cdot 8$ days. This difference reflects our policy of delaying the introduction of enteral feeding until babies are out of intensive care. It is important to note, however, that the 17 babies who developed necrotising enterocolitis were first fed at a mean age of 10 days which is significantly later than the rest of the non-treatment group who escaped necrotising enterocolitis. Only a prospective randomised trial can decide whether it is the use of vancomycin or the delaying of enteral feeds (or indeed some other factor) that was responsible for preventing necrotising enterocolitis in our treatment group.

An inevitable worry associated with the widespread prophylactic use of any antibiotic is that of inducing resistance in bacteria. Vancomycin has a remarkable reputation for not doing so, and it has been in use for a long time. Further investigation of this would be desirable if vancomycin prophylaxis in the very low birthweight baby was to be generally adopted.

\footnotetext{
References

${ }^{1}$ Egan EA, Gonzalo Mantilla, Nelson RM, Eitzman DV. A prospective controlled trial of oral kanamycin the prevention of neonatal necrotizing enterocolitis. J Pediatr 1976;89:467-72.

2 Boyle R, Nelson JS, Stonestreet BS, Peter G, Oh W. Alterations in stool flora from oral kanamycin prophylaxis of necrotizing enterocolitis. J Pediatr 1978;93:861.

${ }^{3}$ Grylack LJ, Scanlon JW. Oral gentamicin therapy in the prevention of neonatal necrotizing enterocolitis. Am J Dis Child 1978;132:1192-4.

${ }^{4}$ Brantley V, Hiatt L, Hegyi T. Effectiveness of oral gentamicin
} 
in reducing the incidence of necrotizing enterocolitis in treated and control infants. Pediatric Research Abstracts 1980;14:1001.

5 Han VKM, Sayed H, Chance GW, Brabyn DG, Shaheed WA. An outbreak of clostridium difficile necrotizing enterocolitis: a case for oral vancomycin therapy? Pediatrics 1983;71:935-41.

${ }^{6}$ Eyal F, Sagi E, Arad I, Avital A. Necrotising enterocolitis in the very low birthweight infant: expressed breast milk feeding compared with parenteral feeding. Arch Dis Child 1982;57: 274-6.

Correspondence to Dr PRF Dear, Department of Paediatrics and Child Health, St James's University Hospital, Beckett Street, Leeds LS9 TTF.

Accepted 27 June 1988

\title{
Congenital syphilis in the newborn
}

\author{
V CHAWLA, P B PANDIT, AND F K NKRUMAH \\ Department of Paediatrics and Child Health, University of Zimbabwe, Harare, Zimbabwe
}

SUMMARY We studied 53 newborn babies with congenital syphilis. The common clinical features seen were low birth weight, hepatosplenomegaly, anaemia, jaundice, and symmetrical superficial desquamation of the skin affecting palms and soles. The presence of these clinical signs is highly suggestive of early congenital syphilis. Hydrops fetalis without rhesus or $\mathrm{ABO}$ isoimmunisation should always arouse the suspicion of congenital syphilis.

Sexually transmitted diseases continue to be a serious public health problem all over the world. In recent years an increase in the incidence of acquired and congenital syphilis has been reported from both developed and developing countries. ${ }^{1}$ The present study, which emphasises the neonatal aspects of this disease, was undertaken to document the clinical range of congenital syphilis in the neonatal period, identify common clinical features of the disease in this age group, and assess the immediate prognosis of the disease in the neonatal period.

\section{Patients and methods}

Newborn babies included in the study were born in the greater Harare area and admitted to the neonatal unit, Harare Central Hospital, which is the referral centre for all high risk newborn babies in the city of Harare, Zimbabwe. The total number of newborns admitted to the neonatal unit during the study period, 1 January to 31 December 1986, was 5287 .

The diagnosis of early congenital syphilis was based on positive serology for syphilis in mother or infant, or both, in the presence of two or more clinical signs suggestive of early congenital syphilis. ${ }^{2}$ The clinical signs regarded as suggestive of early congenital syphilis were as follows: (a) haematological manifestations - that is, anaemia, thrombocy- topenia; (b) hepatomegaly or hepatosplenomegaly; (c) jaundice; (d) mucocutaneous lesions-for example, snuffles, condylomata lata, skin rash, peeling skin of palms and soles, etc; (e) nonimmune hydrops; (f) Parrot's pseudoparalysis; and (g) radiological appearance in the long bones suggestive of congenital syphilis.

The serological tests employed for establishing the diagnosis of congenital syphilis were the rapid plasma reagin test and Treponema pallidum haemagglutination assay. A total of 53 newborn babies fulfilled the diagnostic criteria laid down above and were included in the study.

\section{Results}

The frequency of clinical features at presentation is shown in the table. Hepatomegaly or hepatosplenomegaly of $2 \mathrm{~cm}$ to $6 \mathrm{~cm}$ was present in $28(53 \%)$ and $26(49 \%)$ patients respectively. Clinical jaundice was noted in 25 infants $(47 \%)$. Unconjugated hyperbilirubinaemia was much more common than

Table Frequency of clinical features in 53 newborn babies with congenital syphilis

\begin{tabular}{ll}
\hline Clinical feature & No (\%) \\
\hline Low birth weight & $41(77)$ \\
Hepatomegaly & $28(53)$ \\
Splenomegaly & $27(51)$ \\
Hepatosplenomegaly & $26(49)$ \\
Anaemia* & $16(50)$ \\
Thrombocytopenia & $12(38)$ \\
Jaundice & $25(47)$ \\
Skin lesions & $20(38)$ \\
Respiratory distress & $10(19)$ \\
Hydrops fetalis & $3(6)$ \\
Snuffles & $2(4)$ \\
Pseudoparalysis & $2(4)$
\end{tabular}

${ }^{*}$ Haemoglobin concentrations and platelet counts were available in 32 babies only, therefore percentages are from a total number of 32 . 